\title{
Dynamics of Quantum-Dot Mode-Locked Lasers With Optical Injection.
}

\author{
Tatiana Habruseva, Student Member, IEEE, Guillaume Huyet, Senior Member, IEEE, and \\ Stephen P. Hegarty, Member, IEEE
}

\begin{abstract}
We present the dynamics of quantum-dot passively mode-locked semiconductor lasers under optical injection. We discuss the benefits of various configurations of the master source including single, dual and multiple coherent frequency source. In particular, we demonstrate that optical injection can improve the properties of the slave laser in terms of time-bandwidth product, optical linewidth and timing jitter.
\end{abstract}

Index Terms-Quantum-dot lasers, phase locked oscillators, mode locked lasers, optical injection.

\section{INTRODUCTION}

Q Uantum-dot (QD) monolithic mode-locked laser diodes (MMLDs) grown on GaAs substrates have been the subject of much research interest in the last number of years, with excellent results obtained in terms of pulsewidth, timebandwidth product and repetition rate. For a review, please see [1]. Some of the early interest in QDs as a mode-locking material were motivated by the wide spectral gains of QD layers, a consequence of the inhomogeneous broadening of StranskiKrastanov grown QDs. Had these wide spectra been coherently combined and dechirped, then pulsewidths approaching $100 \mathrm{fs}$ could have been generated. Typically however, mode-locked emission is restricted to fraction of this wide bandwidth, but QD-MMLDs have revealed different characteristics that explain their excellence in this application. Comparatively low Stark shift, low density of states giving high saturation powers and low saturation fluences, and ultrafast carrier dynamics have led to both rapid photocarrier sweepout [2], [3] and rapid refilling of ground-state electronic levels from electrons stored in the QD excited states [4]. This swift (order picosecond) gain recovery has not been reported in pump-probe measurements of quantum-dash (QDash) mode-locked lasers grown on InP substrates, which can be attributed to the much reduced electronic confinement in these nanostructures [5].

For InAs/GaAs QDs, a significant portion of devices make use of p-doped active regions. This technique combats the poorer confinement for holes in the QDs by adding an excess of holes close to the QD layer, often employing doping with carbon. The benefits for $\mathrm{CW}$ lasers are clear, with higher modal gains making shorter cavities possible, necessary to operate the laser directly modulated up to $10 \mathrm{GHz}$ [6]. Additionally, p-doped CW QD lasers have excellent $T_{0}$ performance as the excess doping inhibits the gain reduction with increased hole

T. Habruseva, G. Huyet and S.P. Hegarty are with the Center of Advanced Photonics and Process Analysis, Cork Institute of Technology and Tyndall National Institute, Cork, Ireland.

Manuscript received escape [7]. For mode-locked lasers however, these aspects of active region doping are not necessarily beneficial. QDMMLDs can be very long, approaching $1 \mathrm{~cm}$ in cavity length, removing some of the motivation for higher modal gain. This can be true even for very high repetition rate lasers, with several reports detailing harmonic mode-locking [8] of long devices and the reconfigurability of individually addressable multiple section devices [9]. The additional free-carrier absorption of the doped layers leads to higher threshold currents and reduced laser power, and can be expected to reduce the saturation power. The $T_{0}$ improvement with doping may be useful in certain MMLD applications, but for applications that rely upon a stable repetition rate or spectrum, active temperature control of the laser will be a requirement.

This paper aims to analyse the effect of optical injection in both undoped and p-doped QD MMLDs. As previously shown for p-doped devices [10], we observe a strong reduction of the time-bandwidth product (TBP) due to optical spectral narrowing along with unchanged pulsewidth with injection. Simulations and direct electric field measurements made in [11] have shown that this optical spectrum narrowing can be associated with suppression of waveform instabilities in injection-locked devices.

We have shown previously that when the laser is subjected to optical injection of two mutually coherent frequencies we observe a reduction of the timing jitter, modal optical linewidth and RF linewidth along with TBP improvement [10]. In this paper we further develop the use of dual-tone master sources not restricted to the slave laser repetition rate, and we introduce an additional boundary condition with triple-tone injection, showing the flexibility of the technique. Additionally, we applied the technique to both p-doped and undoped QDMLLs and found similarities in response of the slave for both types of the devices. Finally, injection locked and free-running QD-MLLs are used as master sources showing successful entrainment of QD-MLL slave laser. We show that the locking mechanism is robust versus the addition of extra boundary conditions from the master spectrum and both repetition rate entrainment and phase locking can be achieved with complex master spectra.

\section{EXPERIMENTAL SETUP}

The passively mode-locked lasers (PMLLs) used as slave lasers in the studies were two-section monolithic devices with InAs/GaAs quantum-dot active regions (both p-doped and undoped) with the characteristics as shown in Table 1. Similar devices are described in detail in [12]. 


\begin{tabular}{|l|l|l|l|l|}
\hline Device & Length & Absorber & $F_{r e p}(\mathrm{GHz})$ & Doping \\
\hline$P_{1}$ & 4.3 & $10 \%$ & 9.4 & p-doped \\
\hline$P_{2}$ & 4.3 & $12 \%$ & 9.4 & p-doped \\
\hline$P_{3}$ & 4.3 & $17 \%$ & 9.4 & p-doped \\
\hline$U_{1}$ & 4.0 & $20 \%$ & 10.2 & undoped \\
\hline$U_{2}$ & 4.0 & $20 \%$ & 10.2 & undoped \\
\hline \hline
\end{tabular}

The absorber section shows the absorber length related to the total length in $\mathrm{mm}$ of the laser. After fabrication the devices were cleaved with no coating applied to the facets and mounted on a temperature controlled stage at $19.5^{\circ} \mathrm{C}$. The lasers emitted pulses of a few picoseconds duration at 1.3 $\mu \mathrm{m}$ with a repetition rate of $9.4 \mathrm{GHz}$ (for p-doped) and 10.2 $\mathrm{GHz}$ (for undoped).

The optical linewidth of the devices varied from 10 s to $100 \mathrm{~s}$ of $\mathrm{MHz}$, as shown in Fig. 11 (black) for the undoped laser $U_{2}$. The modal linewidth was minimal at the middle of the optical spectrum increasing to the edges by a quadratic law, with the slope of the parabola proportional to the RF linewidth (see [13]). The red line on Fig. 11 shows the parabolic fit to the measured data. The minimal optical linewidth decreased with current increase [14], and increased with reverse bias. The timing jitter of the devices, integrated over the range of $20 \mathrm{kHz}-80 \mathrm{MHz}$, was on the order of a few ps-10s of ps, depending on the bias conditions.

Three different types of master source were used to perform studies of optical injection locking. Fig. 1 shows the experimental setup of master-slave configurations used in the study. In Section III we focus on the outcomes of the singletone $\mathrm{CW}$ injection of PMLLs. For these experiments we used a commercial tunable laser source (TLS) with a narrow linewidth of $\sim 100 \mathrm{kHz}$ as the master source (Fig. 1(a)).

In Section IV we present studies of the injection locking by a dual-tone coherent master source and in Section V via tripletone injection. To generate the coherent tones, the TLS light was amplitude modulated using a Mach-Zehnder modulator driven via an amplified Rohde\&Schwarz SMR 60 signal generator (Fig. 1(b)). For dual-tone injection, the modulator was biased at the transmission minimum giving two coherent sidebands, with a suppressed carrier frequency, while for triple-tone injection, the carrier frequency had approximately the same amplitude as the sidebands. The measured optical spectra are shown in Fig. 2. The frequency of modulation was set to $f_{r e p} / 2$ for dual-tone injection of two adjacent modes (Fig. 2(a)), to $f_{r e p} * 3 / 2$ for dual injection skipping some modes (Fig. 2(b)), and to $f_{r e p}$ for triple-tone injection (Fig. 2(c)). The measurement of the master signal phase noise was limited by the $1 \mathrm{kHz}$ ESA resolution bandwidth (RBW), at $-20 \mathrm{~dB}$ this width was $4 \mathrm{kHz}$.

In Section VI we describe injection-locking by a multipletone source using a similar PMLL as the master laser (Fig. 1(c)). The light from the gain section of the master PMLL was coupled through the polarisation maintaining (PM) lensed fiber for the injection, while emission from the second master facet was coupled via two lenses and an isolator and used to measure master emission.

For all configurations, the master emission was passed through a PM optical circulator and coupled to the gain section of the slave PMLL via PM lensed fiber. The output of the slave laser from port 3 of the circulator was characterised using optical and electrical spectrum analysers (OSA and ESA respectively).

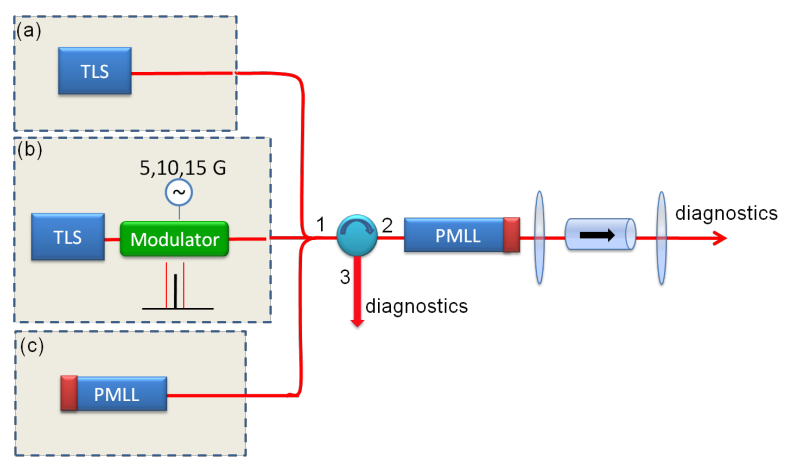

Fig. 1. Experimental setup for single-tone (a), dual- and triple-tone (b) and multiple-tone (c) optical injection.

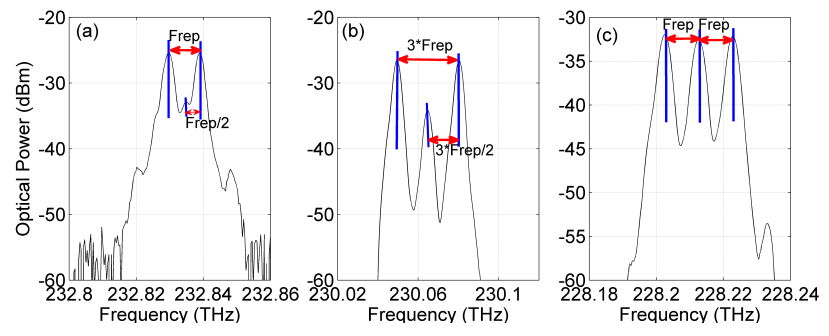

Fig. 2. Optical spectra of master source for dual-tone (a,b) and triple-tone (c) injection.

\section{SingLE-TONE INJECTION}

In this Section we discuss the effect of a single-tone optical injection on the QD-MMLD.

When the device was injected within the locking range of one of its resonator modes the slave repetition rate, optical spectrum, power, timing jitter and phase noise were affected. The injection locking resulted in significant optical spectrum narrowing (bandwidth at $3 \mathrm{~dB}$ was 10-20 times smaller compared to free-running case) and a shift of the laser repetition rate on 10s-100s of MHz. Fig. 3 shows three different regimes observed for the injected laser: the slave can be mode-locked and phase-locked to the injection seed (grey), the slave can be single-mode (red) and the slave can be mode-locked and unlocked from the master (white). The locking range increased with the injection power achieving several $\mathrm{GHz}$ range (as shown in Fig. 3 (grey)); similarly to that observed for multimode [15] and DFB [16] QD lasers. However, an increase of injection power resulted in optical spectrum narrowing and pulses broadening. Fig. 5 shows pulse shapes (solid) and phases (dashed) obtained by direct electric field measurement for different injection powers. We used the frequency-resolved Mach-Zehnder gating EG 130 from Southern Photonics for direct electric field measurement. A similar technique with electro-absorption gating is described in [17]. Finally, at high 


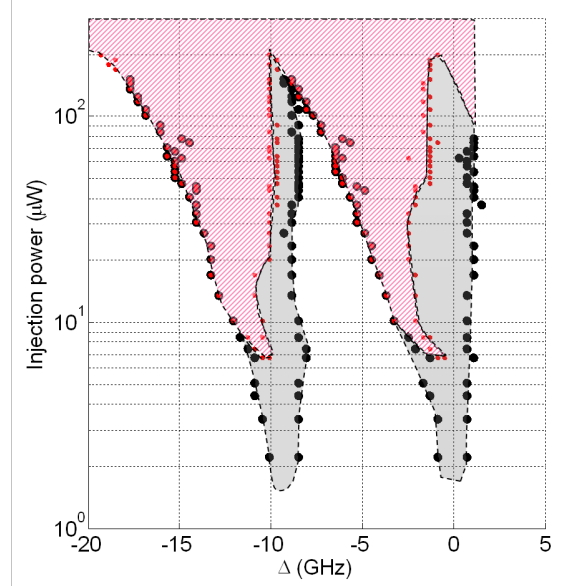

Fig. 3. Injection-locking regions diagram measured by variation of the gain current of the slave laser $P_{1}$ and master power. Gain current: $70-72 \mathrm{~mA}$; absorber bias: $-2 \mathrm{~V}$.

injection power, the device operated only in a single-mode regime and mode-locking was halted (red).

At small injection powers significant optical spectrum narrowing did not visibly affect the pulsewidth, so that autocorrelation traces were similar for injection locked and freerunning laser. This resulted in TBP improvement from $6-10$ when free-running (black) down to 0.4 with injection (red) as shown on Fig. 4. TBP was calculated as a product of 3 $\mathrm{dB}$ optical spectrum bandwidth and pulsewidth measured from autocorrelation trace assuming gaussian pulse shape.

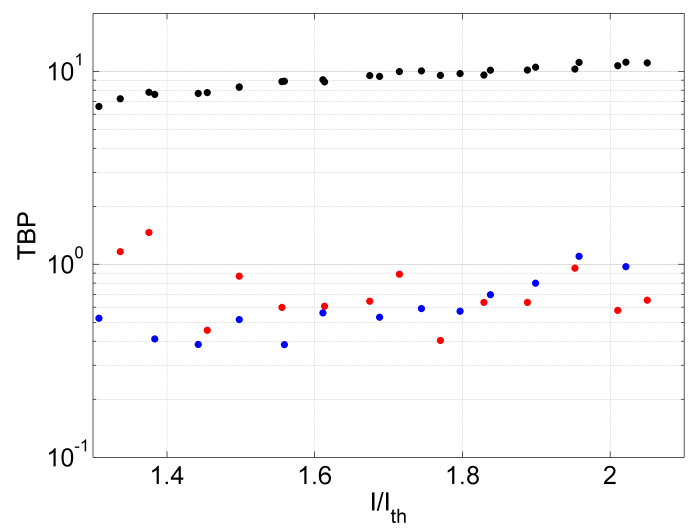

Fig. 4. Time-bandwidth product of laser $P_{3}$ when free-running (black), with single-tone (red) and dual-tone (blue) injection. Absorber bias: $-2 \mathrm{~V}$.

Fig. 6 shows the optical spectra (c) and individual modal linewidths for a free-running PMLL (a) and with single-tone optical injection (b), red. The linewidth was measured by heterodyning with a narrow linewidth TLS.

The single-tone injection greatly reduced the phase noise of the device, so that the optical linewidth was reduced to $0.1-30$ $\mathrm{MHz}$ for the injected laser compared to $200-800 \mathrm{MHz}$ for the free-running case. Several modes close to the injection wavelength were phase-locked to the master (that is, they

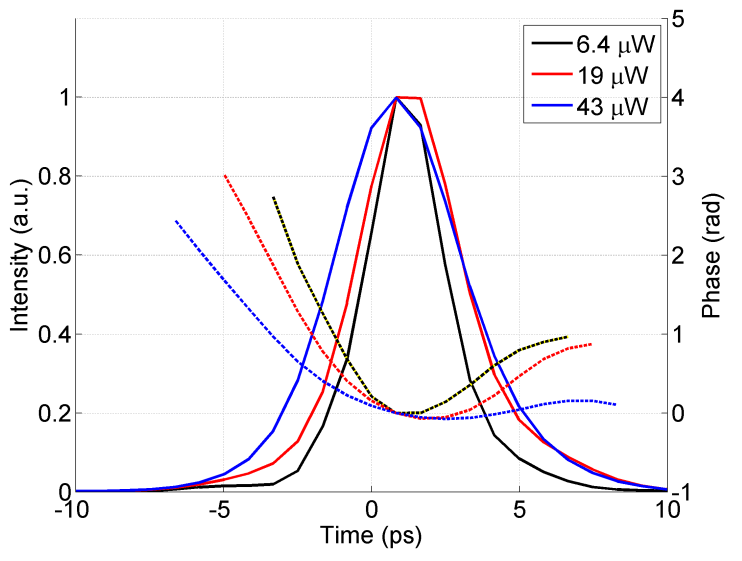

Fig. 5. Pulses (solid) and phases (dashed) for different injection powers. Laser $P_{2}$; gain current: $104 \mathrm{~mA}$; absorber bias: $-6 \mathrm{~V}$.

attained the narrow linewidth of the master TLS). However, far from the injection wavelength the modal linewidth increased. Single-tone injection did not control the repetition rate of the device and in fact could lead to an increase of timing jitter [18].
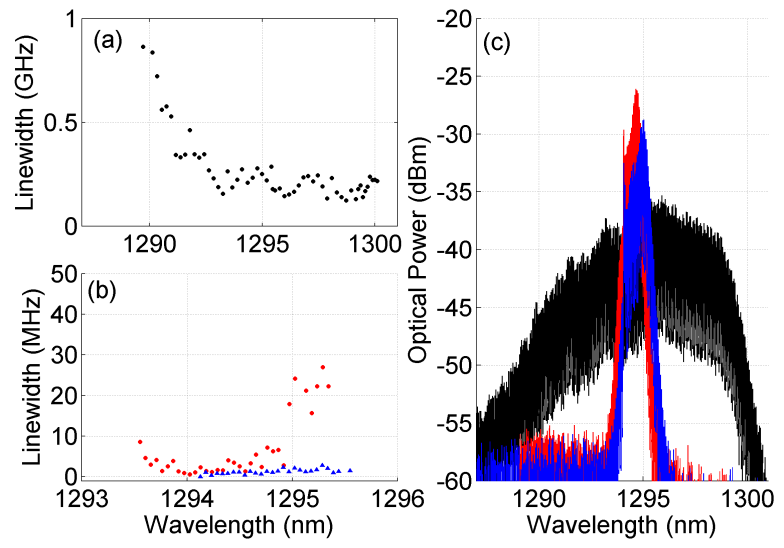

Fig. 6. Optical modal linewidth of free-running PMLL (a), PMLL with single-tone (b, red) and dual-tone (b, blue). (c) Corresponding optical spectra for free-running laser (black), with single-tone (red) and dual-tone (blue) injection. Laser $P_{1}$; gain current: $109.6 \mathrm{~mA}$; absorber bias: $-3.8 \mathrm{~V}$.

Thus, further efforts should be made to reduce the noise, control the repetition rate of the device and achieve a highly coherent frequency comb. In particular, via injection locking to a two-tone coherent master source [19], or combination of single-tone $\mathrm{CW}$ injection and hybrid mode-locking.

\section{DUAL-TONE INJECTION}

The effect of the dual-tone optical injection upon the pdoped QD lasers was described previously in [10]. There, we showed that dual-tone injection resulted in optical spectrum narrowing and tuning, optical linewidth narrowing and jitter reduction for the p-doped laser. Fig. 4 shows TBP improvement with dual-tone injection (blue) compared to a free-running regime (black).

When the slave was locked to a dual-tone coherent source its RF linewidth of the first harmonic was reduced greatly, below 
$1 \mathrm{kHz}$ RBW of the ESA, with a $-20 \mathrm{~dB}$ bandwidth of $4 \mathrm{kHz}$. This instrument limited spike sat above a phase noise pedestal, which led to the measurable jitter of the slave. The timing jitter was calculated via integration of the phase noise of the photocurrent signal of the slave first harmonic in the range of $20 \mathrm{kHz}-80 \mathrm{MHz}$. The reduction of integrated timing jitter is shown on Fig. 7. With dual-tone injection the integrated timing jitter of the device was reduced from $11-21$ ps for free-running regime down to $1 \mathrm{ps}-300 \mathrm{fs}$. Similar benefits are also obtained with the undoped laser. With dual-tone injection all measurable linewidths of the modes in the slave's optical spectrum (Fig. 6(c), blue) were reduced down to the master source linewidth with an accuracy of chosen RBW of ESA (300 kHz) (Fig. 6(b), blue).

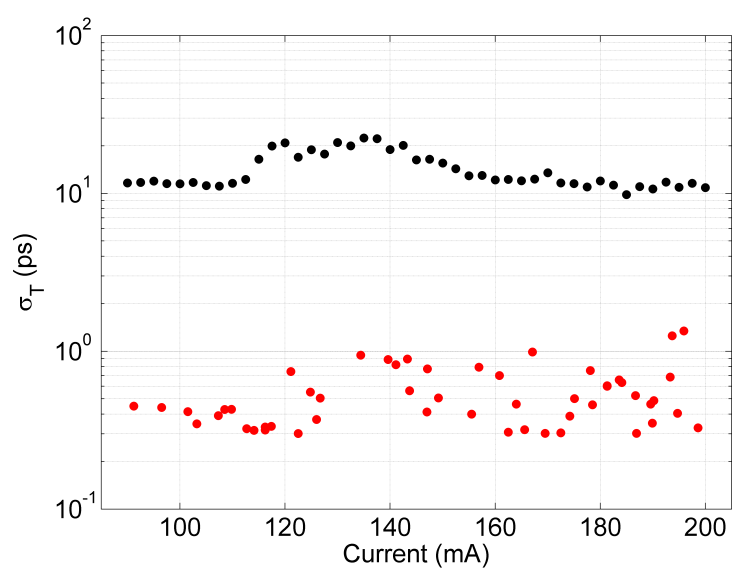

Fig. 7. Integrated timing jitter of free-running PMLL (black), and with dual-tone injection (red). Laser $P_{2}$; absorber bias: -6 V. Reprinted from [10].

Dual-tone coherent master emission allowed tuning of the slave PMLL repetition rate over a range of $25 \mathrm{MHz}$ with a great jitter reduction, as shown in Fig. 8, where $F_{2}$ is the separation between the two coherent master lines.
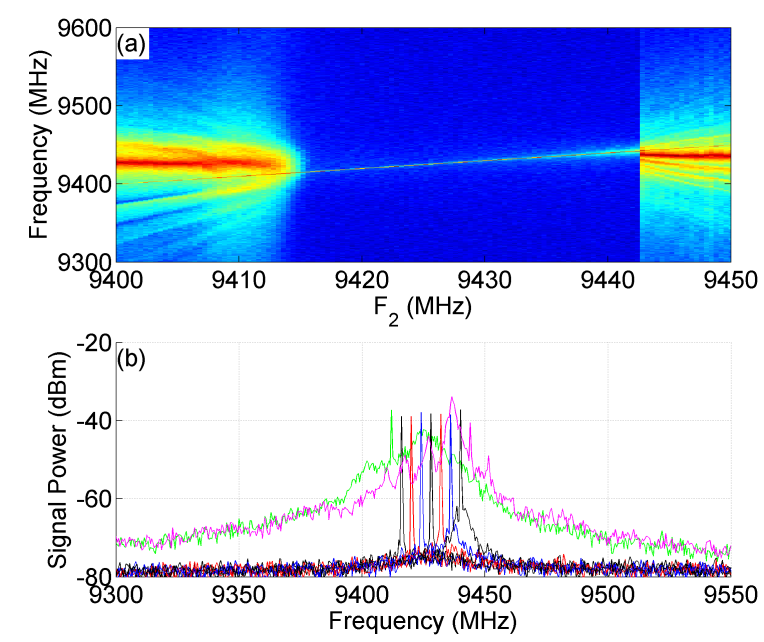

Fig. 8. (a) Evolution of the slave PMLL electronic spectrum with modulator frequency detuning. (b) RF signal of injected PMLL for different modulator frequencies. Laser $P_{2}$; gain current: $100 \mathrm{~mA}$; absorber bias: $-6 \mathrm{~V}$.
The $f_{\text {rep }}$ tuning range increased with optical injection power and saturated at small injection powers of $\sim 8 \mu W$ as shown in Fig. 9. Thus, we can still be in the range of low injection powers and achieve large frequency tuning.

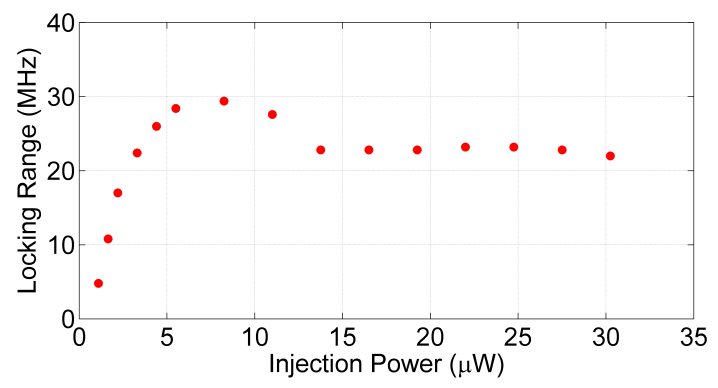

Fig. 9. Locking range of the slave frequency detuning as a function of the injected optical power. Laser $P_{2}$; gain current: $100 \mathrm{~mA}$; absorber bias: $-6 \mathrm{~V}$.

In order to understand the locking mechanism, different separations between the injected sidebands $\left(F_{2}\right)$ were used. We set the signal generator at $\sim 15 \mathrm{GHz}$, which corresponds to $F_{2} \sim 3 * f_{\text {rep }}$ (Fig.2(b)). Similar to the injection of adjacent modes, dual injection with skipped modes still allowed jitter and linewidth reduction as well as optical spectrum narrowing and large frequency tuning. Fig. 10 shows RF signals of the first harmonic for the free-running laser (black) and the injected laser (red). The RF linewidth was reduced significantly, below $1 \mathrm{kHz}$ RBW of the ESA. If we consider the locking mechanism with dual-tone injection as a result of FWM terms arising across the slave spectrum, skipping two modes between injected frequencies should result in no optical linewidth decrease for some slave modes, whose frequencies are not arithmetic combinations of the two master frequencies. In the experiment the modal linewidth of all the slave modes was narrowed. The inset shows heterodyne beating signals of several adjacent modes. This indicates that the FWM terms generated by the two frequency source are not sufficient to describe the overall spectral properties of the slave laser.

\section{TRIPLE-TONE INJECTION}

Dual-tone injection shows a significant improvement of PMLL characteristics, but it requires to operate the modulator with suppressed carrier frequency. Without this constraint the modulator generates additional frequencies. In Fig. 2(c) the modulator bias was set so that the carrier frequency had approximately the same amplitude as the sidebands. Fig. 11 and 12 show the results obtained for PMLL $U_{2}$ using such triple-tone injection.

In this configuration we reproduce the benefits observed with dual-tone injection, including optical spectrum narrowing, modal linewidth reduction (Fig. 11, blue), improvement in the timing jitter, and RF frequency tuning (Fig. 12). Remarkably, the introduction of the additional master frequency brought an additional phase constraint, but did not significantly alter the locking range of the laser (Fig. 12) enabling the possibility of using a multiple-tone sources such as PMLL as a master laser. 


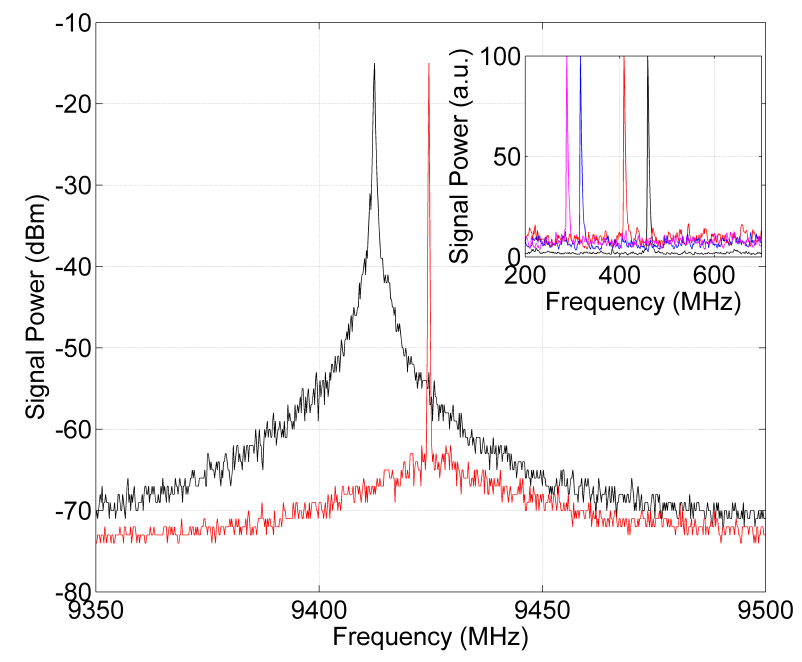

Fig. 10. RF signals of the first harmonic for free-running (black) and injected laser (red). Inset: heterodyne beating signals of different modes of the injected slave laser. Laser $P_{2}$; gain current: $110 \mathrm{~mA}$; absorber bias: $-6 \mathrm{~V}$.
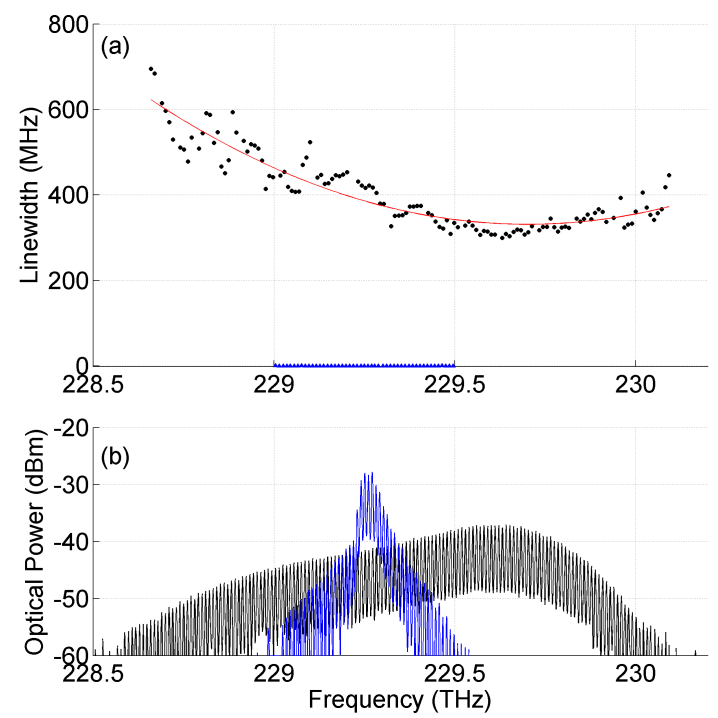

Fig. 11. Modal linewidth of free-running (black) and triple-tone injected (blue) laser $U_{2}$. (b) Corresponding optical spectra.

\section{MASTER-SLAVE}

In this Section we discuss the configuration where a PMLL was used a master source. In order to stabilise master emission, the master laser was dual-tone injected as shown in Fig. 13. Two coherent sidebands with separation close to the master repetition rate were injected to port 1 of the PM circulator and coupled from port 2 to the gain section of the master laser through the PM lensed fiber. Port 3 was coupled to the gain section of the slave laser and port 4 went to diagnostics. The master emission was characterised using the second master laser facet. This dual-tone injection led to optical spectrum narrowing of the master as well as reduced master jitter and modal linewidth.

The optical and electronic spectra of the slave laser when free-running (black) and injected master laser (blue) are shown

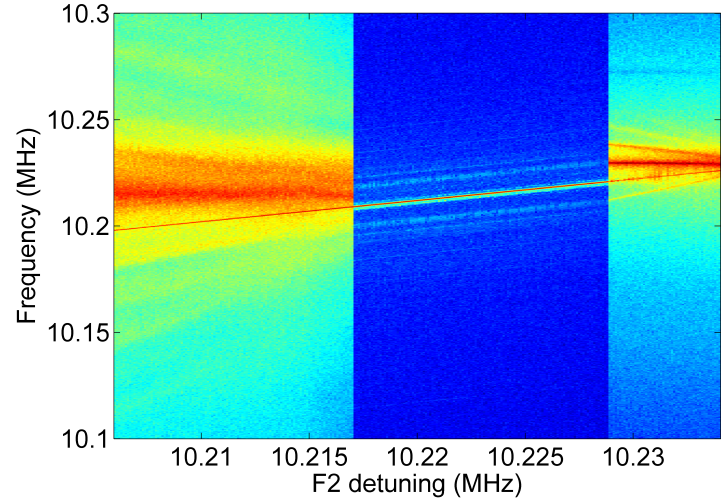

Fig. 12. Intensity graph of slave electronic spectrum evolution with sidebands detuning for triple-tone injection. Laser $U_{2}$; gain current: $67 \mathrm{~mA}$; reverse bias: $-3 \mathrm{~V}$.

in Fig. 14 (a) and (b). When the slave, $U_{1}$, was locked to the injected master, $U_{2}$, its optical spectrum was narrowed, nearly reproducing the master (Fig. 14(c), red) and the timing jitter was reduced to that of the master source $((d)$, red).

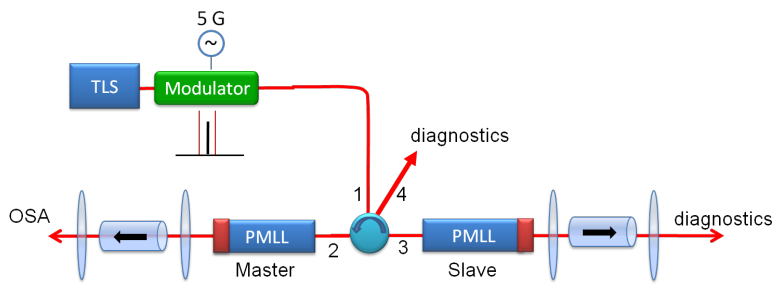

Fig. 13. Experimental setup for optical injection using dual-tone injection locked PMLL as a master source.

The modal linewidth of the slave was reduced greatly, down to the RBW, demonstrating the phase-locking of the slave to the master. Beating signals of heterodyne linewidth measurements for different slave modes across the spectrum are shown in Fig. 15.

One exploitation path is to lock a noisy slave laser to a master source with better performance (for instance, hybridly mode-locked). To synchronise two PMLLs their spectral characteristics must be similar and their repetition rates within the locking range. In the case we discuss now the master laser has a lower timing jitter than that of the slave? which will be transferred.

Fig. 16 shows the output of the slave locked to the master ((c),(d), red) and the initial spectra of the master (blue) and the slave (black). The injected slave optical spectrum ((c), red) was close to its initial ((a), black) with some additional amplified modes due to injection locking. However, the slave repetition rate was tuned to the master $((\mathrm{d})$, red) with a reduction of the timing jitter due to less noisy master signal ((b),(d), blue).

To study the mutual coherence of master and slave the setup shown in Fig. 17 was used. The output of the master laser was passed through a polarisation controller and a tunable delay line and coupled using a 50/50 fiber coupler with the slave laser output shifted by $80 \mathrm{MHz}$ via an acousto-optic modulator. We used the tunable delay to adjust the pulse arrival time of 

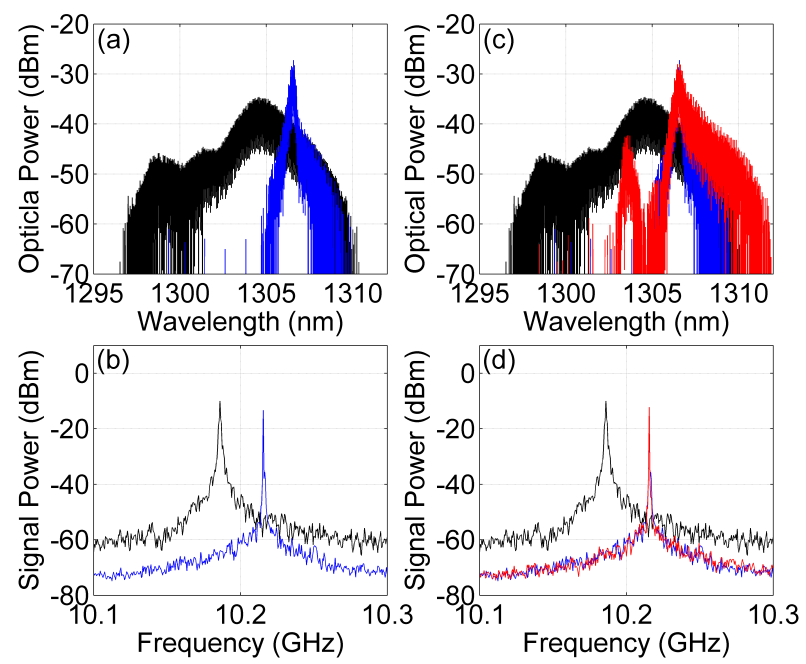

Fig. 14. (a),(c) Optical spectra of the dual-tone injected master laser $U_{2}$ (blue), the slave laser $U_{1}$ when free-running (black), and the slave laser locked to the injected master (red); (b),(d) The RF spectra of the dual-tone injected master (blue), the slave when free-running (black), and the slave laser locked to the master (red). Master laser: $U_{2}$; gain current: $71 \mathrm{~mA}$; absorber bias: -3.3 V. Slave laser: $U_{1}$; gain current: $134 \mathrm{~mA}$, absorber bias: $-7 \mathrm{~V}$.

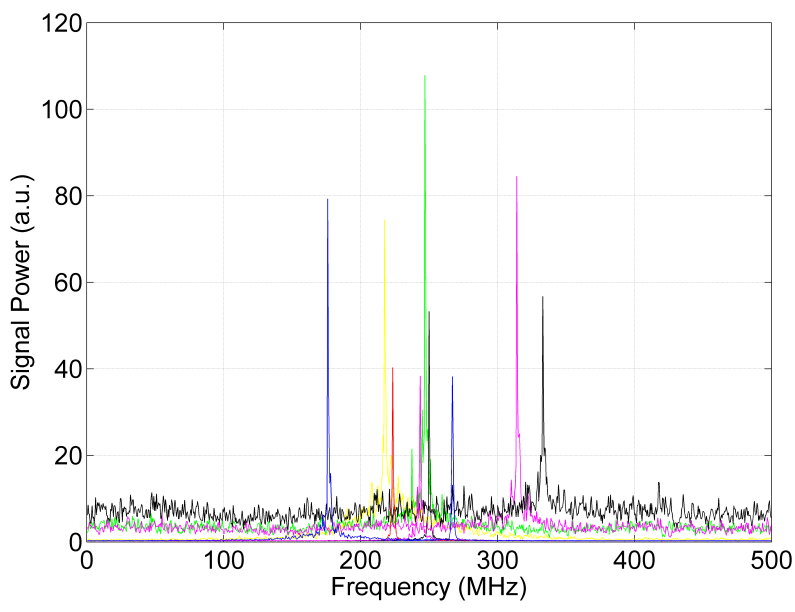

Fig. 15. Heterodyne beating signals of slave linewidth measurements for different slave modes across the spectrum.

the master and slave, and optionally a $10 \mathrm{~km}$ fiber spool that exceeded the coherence length of the lasers. The beating signal at $80 \mathrm{MHz}$ was measured using an ESA.

Without injection from the master there was no beating at $80 \mathrm{MHz}$ observed as the lasers were independent and had different wavelengths. Injection-locking of the the slave to the master resulted in the appearance of a beating tone at $80 \mathrm{MHz}$ observed on the ESA. The beating signals are shown in Fig. 18. With the long delay in one of the branches (see Fig. 17, dashed line) the outputs of the master and the slave were not coherent and the beating signal linewidth (FWHM) was around $30 \mathrm{MHz}$ (Fig. 18,(a)); however, without the long delay and adjusting the short delay to overlap pulses from the master and slave outputs, the beating signal linewidth was reduced to the RBW demonstrating the mutual coherence of the devices (Fig. 18,(b)).
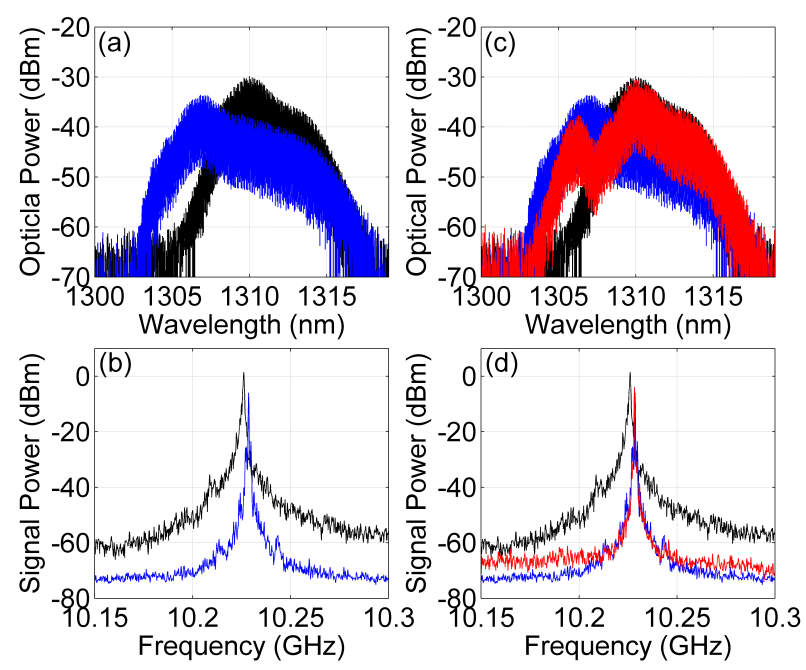

Fig. 16. (a),(c) Optical spectra of the master (blue), the slave when freerunning (black), and the slave laser locked to the master (red); (b),(d) The first harmonic RF spectra of the master (blue), the slave when free-running (black), and the slave laser locked to the master (red). Master laser: $U_{2}$; gain current: $120 \mathrm{~mA}$; absorber bias: $-4 \mathrm{~V}$. Slave laser: $U_{1}$; gain current: $122 \mathrm{~mA}$, absorber bias: $-6 \mathrm{~V}$.

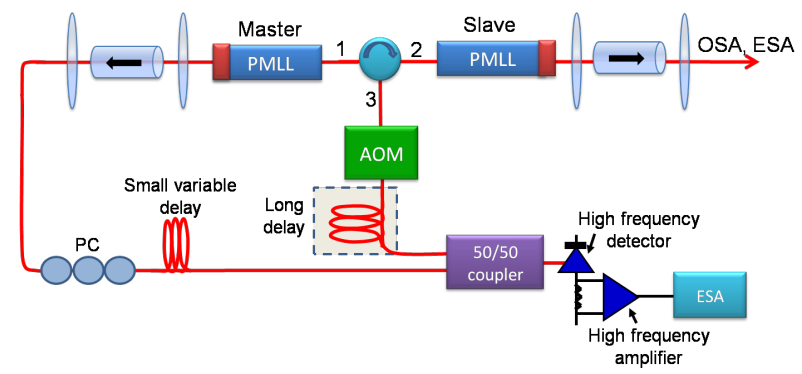

Fig. 17. Experimental setup for checking mutual coherence of the master and slave PMLLs.

The reproduction of the master PMLL optical spectrum and repetition rate by the slave could be used for signal amplification, while the coherence of master and slave sources makes it attractive for coherent communications and wide frequency comb generation [20].

\section{CONCLUSION}

A technique to control and improve InAs/GaAs quantumdot PMLL performance was investigated for both $\mathrm{p}$-doped and undoped active region devices. Single-tone injection resulted in partial narrowing of the modal optical linewidths but could lead to a timing jitter increase. Dual coherent tone injection reduced modal optical linewidths, phase noise and timing jitter of the PMLLs and allowed device repetition rate control over a $25 \mathrm{MHz}$ range at low injection levels, consistent with tuning ranges found in hybridly mode-locked samples, which makes it attractive for optical communications [21], [22]. The relaxation of the pure dual tone constraint with the admission of a third coherent tone did not affect significantly the RF tuning locking range and reproduced the benefits of the dualtone injection. This relaxation is equivalent to the admission of 


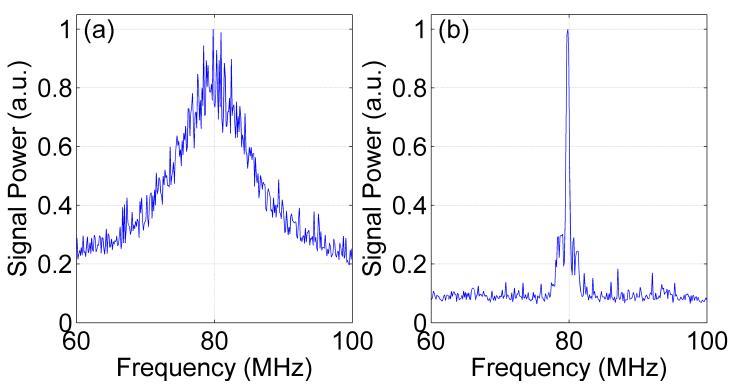

Fig. 18. Self-heterodyne beating signals between master and slave when the slave is locked and slave's output is non-coherent with master's (a), coherent with master's output (b).

significant DC component in the master spectrum, reducing the requirements for modulator power. Finally, a highly complex PMLL was used as a master source and the slave successfully phase locked. The slave laser's repetition rate was locked to the master's with improvement of the slave timing jitter. When the master coherence was improved through locking to a dualtone clock, the slave laser RF linewidth and modal optical linewidths were reduced to the spectrum analyser limits. The mutual coherence of the two PMLLs allows distribution of a comb of highly coherent lines to multiple locations in an optical circuit or indeed to slave lasers separated by a fibre link from the master laser. This cloning of the master coherence makes it attractive for coherent communication applications and wide frequency comb and short pulse generation.

\section{ACKNOWLEDGMENT}

This research was enabled by the Higher Education Authority Program for Research in Third Level Institutions (20072011) via the INSPIRE programme and the authors also gratefully acknowledge the support of Science Foundation Ireland under Contract No. 07/IN.1/I929.

\section{REFERENCES}

[1] E. U. Rafailov, M. A. Cataluna, and W. Sibbett , "Mode-locked quantumdot lasers," Nature Photonics, vol. 1, pp. 395-401, Jul. 2007.

[2] D. B. Malins, A. Gomez-Iglesias, S. J. White, W. Sibbett, A. Miller, and E. U. Rafailov, "Ultrafast electroabsorption dynamics in an InAs quantum dot saturable absorber at $1.3 \mu \mathrm{m}$," Appl. Phys. Lett., vol. 89, no. 17, p. 171111, Oct. 2006.

[3] T. Piwonski, J. Pulka, G. Madden, G. Huyet, J. Houlihan, E. A. Viktorov, T. Erneux, and P. Mandel, "Intradot dynamics of InAs quantum dot based electroabsorbers," Appl. Phys. Lett., vol. 94, no. 12, p. 123504, Mar. 2009.

[4] T. Piwonski, I. O Driscoll, J. Houlihan, G. Huyet, R. J. Manning, and A.V. Uskov, "Carrier capture dynamics of InAs/GaAs quantum dots," Appl. Phys. Lett., vol. 90, p. 122108, Mar. 2007.

[5] S.C. Heck, S. Osborne, S.B. Healy, E.P. O'Reilly, F. Lelarge, F. Poingt, O. Le Gouezigou, A. Accard, "Experimental and Theoretical Study of InAs/InGaAsP/InP Quantum Dash Lasers," IEEE J. Quantum Electron., vol. 45, no. 12, pp. 1508-1516, Dec. 2009.

[6] K. Takada, Y. Tanaka, T. Matsumoto, M. Ekawa, H. Z. Song, Y. Nakata, M. Yamaguchi, K. Nishi, T. Yamamoto, M. Sugawara, and Y. Arakawa, "10.3-Gb/s Operation over a Wide Temperature Range in 1.3- $\mu \mathrm{m}$ Quantum-dot DFB Lasers with High Modal Gain," in OSHA/OFC/NFOEC, 2010, OThK2.

[7] R.R. Alexander, D.T.D. Childs, H. Agarwal, K.M. Groom, H.-Y. Liu, M. Hopkinson, R.A. Hogg, M.Ishida, T. Yamamoto, M. Sugawara, Y. Arakawa, T.J. Badcock, R.J. Royce and David J. Mowbray, "Systematic Study of the Effects of Modulation p-Doping on 1.3- $\mu \mathrm{m}$ Quantum-Dot Lasers,"J. Quantum Electron., Vol. 43, no. 12, pp. 1129-1139, Dec. 2007.
[8] A.R. Rae, M.G. Thompson, R.V. Penty, I.H. White, A.R. Kovsh, S.S. Mikhrin, D.A. Livshits, I.L. Krestnikov, "Harmonic Mode-Locking of a Quantum-Dot Laser Diode," in 19th Annual Meeting of the IEEE, Lasers and Electro-Optics Society, Montreal, Cabada, Oct./Nov. 2006, pp. 874 $-875$.

[9] Y. C. Xin, Y. Li, V. Kovanis, A. L. Gray, L. Zhang, and L. F. Lester, "Reconfigurable quantum dot monolithic multisection passive modelocked lasers", Opt. Express, vol. 15, no. 12, pp. 7623-7633, Jun. 2007.

[10] T. Habruseva, S. O’Donoghue, N. Rebrova, D. A. Reid, L. P. Barry, D. Rachinskii, G. Huyet, and S. P. Hegarty, "Quantum-dot mode-locked lasers with dual mode optical injection," IEEE Photon. Tech. Lett., vol. 22, no. 6, pp. 359-361, Mar. 2010.

[11] N. Rebrova, T. Habruseva, G. Huyet and S. P. Hegarty, "Stabilization of a passively mode-locked laser by continuous wave optical injection," Appl. Phys. Lett., vol 97, pp. 101105, Sep. 2010.

[12] M.T. Todaro, J.P. Tourrenc, S.P. Hegarty, C. Kelleher, B. Corbett, G. Huyet and J.G. McInerney, "Simultaneous achivement of narrow pulse width and low pulse-to-pulse timing jitter in $1.3 \mu \mathrm{m}$ passively modelocked quantum-dot lasers," Opt. Lett., vol. 31, no.21, pp. 3107-3109, 2006.

[13] F.X. Kartner, U. Morgner, T. Schibli, R. Ell, H.A. Haus, J.G. Fujimoto and E.P. Ippen, "Few-Cycle Pulses Directly from a Laser," Topic Appl. Phys., vol.95, no. 40, pp. 73-136, 2004.

[14] T. Habruseva, S. O’Donoghue, N. Rebrova, F. Kéfélian, S.P. Hegarty and G. Huyet, "Optical linewidth of a passively mode-locked semiconductor laser," Opt. Lett., vol. 34, no. 21, pp. 33073309, Nov. 2009.

[15] D. Goulding, S.P Hegarty. O. Rasskazov, S. Melnik, M. Harnett, G. Greene, J. G. McInerney, D. Rachinskii and G. Huyet, "Excitability in a Quantum Dot Semiconductor Laser with Optical Injection," Phys. Rev. Lett., vol. 98, pp. 153903, Apr. 2007.

[16] B. Kelleher, D. Goulding, S. P. Hegarty, G.Huyet, D.-Y. Cong, A. Martinez, A. Lemaitre, A. Ramdane, M. Fischer, F. Gerschuetz and J. Koeth,"Excitable phase slips in an injection-locked single-mode quantum-dot laser," Opt. Lett., vol. 34, no. 4, pp. 440-442, Feb. 2009.

[17] C. Dorrer and I. Kang, "Simultaneous temporal characterization of telecommunication optical pulses and modulators by use of spectrograms," Opt. Lett., vol. 27, no. 15, pp. 1315-1317, Aug. 2002.

[18] T. Habruseva, S. O'Donoghue, N. Rebrova, S.P. Hegarty and G. Huyet, "Quantum-dot mode-locked lasers with optical injection," SPIE Proceedings, vol. 7608, Jan. 2010.

[19] W. Lee and P.J. Delfyett, "Dual-mode injection locking of two independent modelocked semiconductor lasers," Electron. Lett., vol. 40, no. 19, pp. 1182-1184, Sep. 2004.

[20] W. Lee, H. Izadpanah, R. Menendez, S. Etemad and Peter J. Delfyett, "Synchronized Mode-Locked Semiconductor Lasers and Applications in Coherent Communications," J. Lightwave Technol., vol. 26, no. 8, pp. 908-921, Apr. 2008.

[21] J. Renaudier, B. Lavigne, M. Jourdan, P. Gallion, F. Lelarge, B. Dagens, A. Accard, O. Legouezigou and G.-H. Duan, "First demonstration of all optical clock recovery at $40 \mathrm{GHz}$ with standard-compliant jitter characteristics based on a quantum-dots self-pulsating semiconductor laser," Proc. European Conference and Exhibition on Optical Communications (ECOC), 2005.

[22] M. Costa e Silva, A. Lagrost, L. Bramerie, M. Gay, P. Besnard, M. Joindot, J. C. Simon, A. Shen and G.H. Duan,"Up to $425 \mathrm{GHz}$ all optical frequency down-conversion clock recovery based on Quantum dash Fabry-Perot mode-locked laser," NFOEC, Mar. 2010.

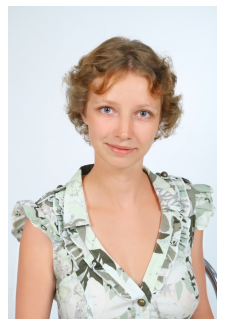

Tatiana Habruseva (M'09) received M.Sc. degree in Laser Physics and Spectroscopy from Belarusian State University, Minsk, Belarus in 2008. She is currently working toward her $\mathrm{PhD}$ degree in Photonic Device Dynamics Group, Cork Institute of Technology and Tyndall National Institute, Cork, Ireland. Her current research interests include modelocked lasers and quantum dot semiconductor lasers. 


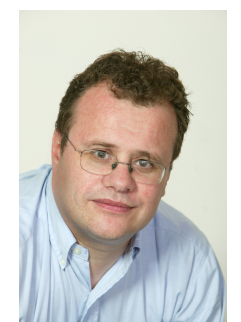

Guillaume Huyet (SM'00) received the M.Sc. degree from ENS Lyon, and the Ph.D. degree from the University of Nice. After postdoctoral positions in Mallorca, Spain, Cork, Ireland, and Glasgow, U.K., he became a Lecturer at University College, Cork, Ireland. In 2002, he received a Science Foundation Ireland Principal Investigator award and became a Senior Research Fellow at University College, Cork. He is currently Science Foundation Ireland Principal Investigator at Tyndall National Institute and Head of Centre at CAPPA, Cork Institute of Technology. His main research interests include nonlinear and quantum optics of semiconductor lasers with a special interest in broad area, external cavity, and quantum dot semiconductor lasers. He has published over 80 papers in international journals.

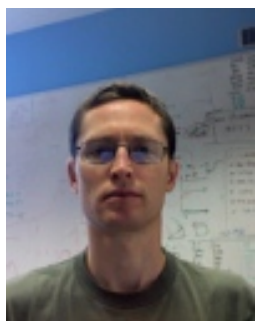

Stephen P. Hegarty (M'04) obtained both the B.Sc. degree in Science and his Ph.D. degree in physics from the University College, Cork. In 1998, he joined Corning Inc., at Corning, New York as a Senior Development Scientist working on novel fiber optical amplifiers for telecommunications. Later he joined Optimight Communications of San Jose as a Senior Optical Engineer, working on ultralonghaul high capacity systems. In 2002, he rejoined University College, Cork as a Senior Researcher. He is now an Science Foundation Ireland Principal Investigator and his major research interests are semiconductor lasers and high-speed systems where he has made significant contributions in both an academic and industrial setting. 